Revue internationale P.M.E.

Économie et gestion de la petite et moyenne entreprise

Revue

internationale

PME

\title{
Cartographie cognitive et accompagnement du créateur d'entreprise
}

\section{Thierry Verstraete}

Volume 10, numéro 1, 1997

URI : https://id.erudit.org/iderudit/1009017ar

DOI : https://doi.org/10.7202/1009017ar

Aller au sommaire du numéro

Éditeur(s)

Presses de l’Université du Québec

ISSN

0776-5436 (imprimé)

1918-9699 (numérique)

Découvrir la revue

Citer cet article

Verstraete, T. (1997). Cartographie cognitive et accompagnement du créateur d'entreprise. Revue internationale P.M.E., 10(1), 43-72.

https://doi.org/10.7202/1009017ar
Résumé de l'article

Cette communication propose une démarche d'accompagnement des créateurs d'entreprise, des dirigeants de TPE, voire de PME. La méthode déployée s'inspire largement d'une proposition faite antérieurement et au centre de laquelle un outil suscitant un intérêt croissant en management stratégique est utilisé : la cartographie cognitive. Cette recherche a une visée opérationnelle et s'appuie pour cela sur une application menée dans une jeune entreprise du secteur de la distribution. Son objet est de tester une démarche en cinq phases pour appréhender l'univers cognitif du créateur relativement à son projet, le sensibiliser au besoin de contrôler les facteurs qu'il identifie comme importants pour l'avenir de son entreprise et l'aider dans la mise en place de systèmes et procédures de contrôle. 


\title{
Cartographie cognitive et accompagnement du créateur d'entreprise
}

\author{
Thierry VERSTRAETE \\ Institut d'administration des entreprises de Lille
}

MOTS CLÉS

\begin{abstract}
Entrepreneuriat - Carte cognitive-Cognition
Création d'entreprise-Accompagnement-Stratégie

Contrôle et audit - Méthode qualitative-PME et TPE - Prospective
\end{abstract}

\begin{abstract}
RÉSUMÉ
Cette communication propose une démarche d'accompagnement des créateurs d'entreprise, des dirigeants de TPE, voire de PME. La méthode déployée s'inspire largement d'une proposition faite antérieurement et au centre de laquelle un outil suscitant un intérêt croissant en management stratégique est utilisé: la cartographie cognitive. Cette recherche a une visée opérationnelle et s'appuie pour cela sur une application menée dans une jeune entreprise du secteur de la distribution. Son objet est de tester une démarche en cinq phases pour appréhender l'univers cognitif du créateur relativement à son projet, le sensibiliser au besoin de contrôler les facteurs qu'il identifie comme importants pour l'avenir de son entreprise et l'aider dans la mise en place de systèmes et procédures de contrôle.
\end{abstract}

\section{L'AUTEUR}

Thierry Verstraete est maître de conférences associé à l'Institut d'administration des entreprises de Lille. II est membre du CLARÉE (Centre lillois d'analyse et de recherche sur l'évolution des entreprises URA CNRS 936) et du GREMCO (Groupe de recherche sur les méthodes de contrôle organisationnel). En outre, il est responsable de l'option "Gestion des petites et moyennes structures de la maîtrise des Sciences de gestion de Lille". Adresse: IAE de Lille, 104, avenue du peuple Belge, 59000 Lille, France. 


\begin{abstract}
This paper proposes a method to accompany the start-up of very small enterprises, or even small and medium-sized enterprises. This method originates from a prior proposal, in which a new strategic management tool is used, namely cognitive mapping. This research has an operational aim, and is based on an application carried out by a young firm in the distribution sector. A five-step process is tested, investigating an individual's cognitive universe relative to his or her start-up project. The individual is made aware of the important factors to be controlled if the firm is to survive and prosper, and is assisted in the implementation of control systems and procedures.
\end{abstract}

\title{
RESUMEN
}

Esta comunicación propone un método de apoyo para los creadores de empresa, los dirigentes de muy pequeñas empresas e incluso de pequeñas y medianas empresas. El método desarrollado se inspira en una propuesta anterior, en la que se empleaba una herramienta de gestión estratégica que suscita un interés creciente: la cartografía cognitiva. Esta investigación tiene una finalidad operativa y por ello se presenta su aplicación a una joven empresa del sector de la distribución. Su objeto es verificar un proceso compuesto de cinco fases para aprehender el universo cognitivo del creador de empresas con respecto a su proyecto, despertarle a la necesidad de comprobar los factores que el identifica como importantes para el porvenir de su empresa y ayudarle en la puesta en marcha de sistemas y procesos de control.

\section{Introduction}

Dans tout entrepreneur, il y a un prospecteur. Nous ne faisons pas ici référence à l'un des types de comportements stratégiques décrit par Miles et Snow, mais à la discipline prospective. Quelles que soient les forces qui animent un individu, il tend à prévoir. Cette prévision est fondée sur les liaisons qu'il fait entre les facteurs qu'il perçoit (éléments, événements, croyances, acteurs, etc.) et à partir desquels il structure ses schèmes cognitifs. Le chef d'entreprise procède ainsi, plus ou moins explicitement, avec ce que nous appellerons les facteurs clés de succès (FCS dans la suite du texte) et les facteurs stratégiques de risque (FSR) ${ }^{1}$. Nombre de démarches lui sont proposées pour identifier ces facteurs (chaîne de valeur, structure du secteur, étalonnage [benchmarking], etc.), démarches pas

1. Henri Bouquin n'est pas étranger à la forte prégnance de la notion de FCS dans notre démarche. Nous lui avons ici emprunté le terme de FSR. S'agissant précisément de terme, chacun y va de son vocable pour désigner ces facteurs (signes vitaux, facteurs stratégiques de l'industrie, etc.). Nous restons fidèle à l'utilisation du terme FCS, nuancé par celui de FSR. Nous avons, ailleurs, développé une conceptualisation de la notion de FCS et de FSR (à paraître). Le lecteur retiendra ici qu'un FCS est une variable essentielle à l'atteinte du futur souhaité par l'entité (un dirigeant, une coalition, 
toujours utilisables par le dirigeant de PME, encore moins par celui de TPE. Dans la synthèse qu'ils font des méthodes d'identification des FCS, Leidecker et Bruno (1984) proposent de combiner les démarches d'essence analytique avec des démarches plus intuitives par l'utilisation de méthodes moins analytiques, comme la consultation d'experts ou la focalisation sur les intuitions et visions d'un individu familier à la firme. Ces démarches peuvent être classées dans un courant dit « heuristique». Piatelli-Palmarini (1995) nous rappelle que « heuristique» partage la même racine que «eurêka », c'est-à-dire le verbe grec qui veut dire «trouver ». « Globalement, les heuristiques sont des stratagèmes mentaux spécifiques qui servent à résoudre des problèmes spécifiques. Une heuristique est une règle simple et approximative - explicite ou implicite, consciente ou inconsciente - qui permet de mieux résoudre une catégorie donnée de problèmes. » (p. 35) En matière de réflexion stratégique, une démarche heuristique se caractérise par la place donnée à l'activation de l'imagination ou de l'intuition, le recours au jugement et à l'expérience, «il est admis depuis longtemps que la gestion stratégique est autant affaire d'intuition et de vision que d'emploi de méthodes quantitatives plus ou moins sophistiquées. » (Desreumaux, 1993, p. 224) «La pensée stratégique est faite de calcul et de jugement » mais ce dernier supplée le premier (Koenig, 1990, p. 18). L'approche rationaliste, dont l'apogée réside dans ce que la planification stratégique a d'extrémiste (Mintzberg, 1994), possède les limites inhérentes aux « capacités cognitives humaines, des coûts élevés de recueil de l'information et de recherche de politiques alternatives, de la non-disponibilité de certains types d'information, de la multitude des variables qu'il faudrait théoriquement prendre en compte, en pratique, il n'est jamais possible d'être totalement exhaustif ni de construire de système hypothético-déductifs complet » (Avenier, 1988, p. 15). En réaction à cet idéal rationaliste, s'est développé un courant « incrémentaliste » dont les préceptes sont de s'instruire à partir de l'action, d'être particulièrement sensible à la mobilisation des hommes et à la surveillance de l'environnement. Mais plutôt que de considérer les deux doctrines comme exclusives, Avenier a montré qu'il était avantageux de les marier, tout comme Desreumaux (1993): « les méthodes analytiques sont précieuses pour structurer les problèmes, mais sans l'aiguillon de l'intuition et le questionnement qu'elle promeut des hypothèses et frontières perceptuelles héritées des techniques d'analyse, le processus de réflexion stratégique risque fort de manquer de créativité

un créateur d'entreprise, voire, avec une tendance organiciste, l'entreprise elle-même). Ce qui différencie le FCS du FSR, c'est qu'il est maîtrisable, alors que le FSR ne l'est pas (par exemple, les conditions météorologiques pour une entreprise agricole) par l'entité considérée (un FSR pour une entité peut être un FCS pour une autre). Malgré cette différence fondamentale, le lecteur nous pardonnera l'utilisation parfois exclusive du terme FCS dans le but de ne pas alourdir le texte. 
entrepreneuriale » (p. 224). Cette créativité entrepreneuriale est sans conteste une dimension prédominante dans la plupart des actes de création d'entreprise et il paraît nécessaire d'utiliser, en matière d'accompagnement, des outils cohérents avec cette dimension.

Nous inscrivons dans les démarches d'essence heuristique la cartographie cognitive comme outil d'identification des facteurs perçus par les acteurs. Nous l'avons utilisée dans une étude opérationnelle portant sur l'accompagnement d'un créateur d'entreprise. Cette étude est l'objet du présent article. Mais, plus largement, la cartographie cognitive est un outil méthodologique de collecte de données pour le chercheur désirant accéder aux représentations des sujets qu'il rencontre et interviewe. Nous légitimerons son utilisation avant de présenter le cadre opératoire de notre étude. Les controverses dont elle fait l'objet résultent de l'utilisation parfois abusive (lorsqu'elle est appelée " au secours » d'un travail méthodologique faible) ou maladroite qui en est faite. Cela lui fait grand tort et le terme de mode est alors employé péjorativement à son propos. Soulignons l'utilisation de techniques fort proches en psychologie, en psychologie sociale, ce que la seule lecture de l'ouvrage coordonné par Abric (1994) permet de constater.

La cartographie cognitive suscite un intérêt croissant en management stratégique, où de plus en plus on reconnaît le rôle de l'intuition et du jugement. Elle est une invitation à la découverte de l'univers cognitif des acteurs en entreprise ${ }^{2}$. En sciences de gestion, elle peut être un mariage subtil entre démarche d'essence analytique et démarche d'essence heuristique, bien qu'elle appartienne fondamentalement à la seconde catégorie. Elle nous semble être un outil pertinent au service de tout un ensemble de problématiques liées à la vision stratégique de l'individu, à l'interprétation de l'environnement, au positionnement de la firme, à l'identification des avantages concurrentiels et des options stratégiques, à l'anticipation et à la prospective, et à l'identification des facteurs clés de succès. En fait, elle est en rapport avec tout ce qui a trait à la perception et à l'interprétation, à l'attention, à la mémoire, à l' apprentissage et à la connaissance, à la résolution de problèmes, à la cognition sociale (Huff et al., 1990) dans le cadre des activités managériales. Une étude de la littérature montre qu'elle est utilisée à des fins multiples. Pour un inventaire de cette documentation, nous renvoyons le lecteur aux ouvrages génériques coordonnés par Axelrod (1976), Huff et al., (1990) et Cossette (1994b), aux numéros spéciaux des revues Journal of Management Studies (mai 1992), Organization Science (août 1994), Revue Française de Gestion (juin-juillet-août 1994), aux textes de Florence Allard-Poesi $(1995,1996)$ ainsi qu'à nos écrits antérieurs.

2. L'inspiration de la psychologie cognitive en management stratégique : voir Vogler (1995, 1996). 
La cartographie cognitive est pour nous un outil à inscrire dans une méthode. Pour cela, des incursions dans d'autres champs disciplinaires sont nécessaires. Après cette légitimation de l'utilisation de l'outil est présentée une application opérationnelle portant sur l'accompagnement du créateur d'entreprise. Il s'agit d'identifier les facteurs que le créateur perçoit comme importants pour la réussite de son projet (les FCS), de le sensibiliser au besoin de recourir à des mesures de contrôle relatives au suivi de ces facteurs puis, lorsque c'est possible, de mettre en place ces mesures. Avant de procéder à cette mise en place, l'accompagnateur apporte son expertise pour mettre au point la vision stratégique du dirigeant. L'objet de cette recherche consiste donc à tester une méthode d'accompagnement du créateur d'entreprise en ce qui concerne la maîtrise de son projet.

\section{Légitimation de l'outil}

\subsection{Origine des cartes cognitives}

Le mot «carte » possède une acception spatiale. Une carte est un support physique représentant un espace géographique sur lequel s'appuie un individu pour s'orienter, estimer des distances, etc. Elle est une représentation graphique fournissant un cadre de référence, un moyen pour décrire le monde et permettre à l'individu de se positionner, de voir où il peut aller (Fiol et Huff, 1992). Par carte cognitive, on entend alors une « représentation intériorisée de l'environnement, de ses propriétés métriques, des relations topologiques entre les sites qui les composent » (Denis, 1989, p. 235). Elle est susceptible de supporter des calculs cognitifs assurant l'orientation de l'individu, calculs dont la validité est déterminée par l'isomorphisme de la carte «à l'égard de l'espace qu'elle représente » et notamment par sa «capacité à conserver les propriétés euclidiennes de l'espace physique » (Ibid.).

La notion de carte cognitive est attribuée à Tolman $(1948)^{3}$. Elle est née d'expériences consistant à placer des rats dans un labyrinthe pour mesurer la représentation mentale ou carte cognitive que l'animal a du labyrinthe en examinant le patron de son parcours, pour analyser et expliquer son comportement spatial et la complexité de sa représentation (Doré et Mercier, 1992). La carte cognitive correspond ici essentiellement à un système de localisation.

3. Il a aussi proposé la notion de «strip map » dans laquelle le système de localisation permettant à l'individu de se déplacer est remplacé par un système de réponses issu d'un apprentissage conditionnant ces réponses, apprentissage ayant, par exemple, la forme suivante : à tel signe tourner à droite, à tel autre tourner à gauche, etc. La structure spatiale n'intervient pas dans les choix de déplacement (Weick et Bougon, 1986, p. 105). 
Parmi les travaux sur l'humain, citons celui de Pailhous (1970) qui utilise comme sujets les chauffeurs de taxis parisiens. Il leur a demandé de tracer de mémoire un plan de la ville de Paris. De son expérience, il tire les conclusions qu'il existe une relation entre performances objectives des chauffeurs et qualité des plans qu'ils savent fournir donc un lien entre performance de l'action et univers des représentations. Il semblerait que deux sortes d'images mentales soient à l'œuvre (Denis, 1989) : 1) une image correspondant à une vue conceptuelle de la ville, sorte de vue aérienne ; 2) une image correspondant à une vue directe et concrète de ce qui se passe au sol.

Ces deux formes de représentation contribuent à la mise en œuvre des «stratégies de déplacements ». Le programme d'actions résulterait de la vue conceptuelle, la vue directe influant sur les «décisions locales». Des biais existent, par exemple, la surestimation des distances pour les trajets comportant des obstacles matériels ou des angles. D'autres types d'erreurs peuvent intervenir dans cette construction mentale spatiale, menant parfois à des incohérences, mais dont les sujets semblent s'accommoder. Elles se rectifient par expérience ambulatoire ou prise de connaissance de cartes géographiques. Ces deux niveaux ont, d'ailleurs, donné lieu à des travaux comparant les processus de construction de la représentation interne de l'environnement spatial par expérience directe, par prise d'informations exécutée sur des substituts figuratifs symboliques (cartes, plans, etc.), voire par écoute d'un discours verbal (l'apprentissage est alors plus long).

Des analogies invitant à des recherches avec le monde décisionnel des gestionnaires sont sans aucun doute possibles. Ainsi, par exemple, l'image relative à la vue conceptuelle peut correspondre à la théorie stratégique du dirigeant, la vue directe de ce qui se passe au sol pouvant être le quotidien qu'il affronte (et où s'expriment la réflexivité et le sens pratique, selon l'expression de Bourdieu), théorie stratégique et quotidien générant l'action. Certes, c'est en partie reconnaître aux individus la possibilité d'agir sur le système dont ils font partie ; c'est aussi reconnaître une part de discrétion managériale dans l'évolution des organisations ou des populations d'organisations. Sans entrer ici dans le débat déterminisme/volontarisme, force est de constater que cela incite à scruter l'univers cognitif des acteurs, car les représentations qu'ils se font du système influencent leurs actions qui, en retour, dans leur déroulement, fournissent des repères perceptifs nécessaires à l'appréhension de leurs propres actions (Livet, 1993). D'ailleurs, l'essai, l'erreur et l'évitement de l'erreur sont des guides cognitifs pour l'action. «L'action se spécifie au fur et à mesure de la découverte des accidents de terrain ou de la reconnaissance de ratés.» (Thévenot, 1993, p. 278)

Les actions ne sont pas neutres et produisent des effets ; elles transforment la réalité que l'auteur interprète et avec laquelle il interagit (Weick, 1979). La 
réflexivité individuelle, élément de la compétence générale d'une personne dans ses rapports à l'univers, peut, à ce titre, être mise en exergue. "Elle est cette capacité que tout être humain a de voir ce qu'il fait en le faisant, et de voir ce que font les autres dans le contexte de son action.» (Audet, 1994, p. 196) En suivant son action, l'individu peut se servir de la « connaissance qu'il a de ce qu'il fait et du contexte dans lequel il le fait pour déterminer le cours de son action» (Ibid.). Le futur que se construit mentalement l'individu comme but (ou non-but) découle de son évolution dans son environnement. Le sujet se forme une idée précise de son but avant et pendant l'action, «la visée de cette idée définit son intention [...] l'action est centrée sur cette visée [...] il [le but] est défini par le resserrement progressif des limites d'un champ de mouvements possibles, et jamais ces limites ne se rejoignent pour définir une et une seule ligne d'action, ni un but ponctuel» (Livet, 1993, p. 293 et 294). Les actions sont conduites dans un milieu social. Or, comprendre la dynamique des interactions sociales d'un individu passe par le repérage de la «vision du monde » qu'il utilise pour prendre position (Abric, 1994). Dans une optique managériale, nous parlerons de la vision stratégique comme d'un sous-ensemble de cette vision du monde.

Scruter l'univers cognitif d'un sujet (un client, un salarié, un dirigeant, etc.) relativement à un objet relève d'un intérêt particulier pour l'expert qui, «muni du référentiel » du sujet, est mieux armé pour le conseiller (conformément au but du sujet). Préalablement à l'apport de son expertise, il lui incombe d'appréhender ce référentiel.

La représentation spatiale n'est qu'un objet d'étude de la cognition humaine; il en existe bien d'autres et le terme de «carte cognitive» est désormais plus largement employé. Dès qu'une modélisation graphique de la cognition est tentée, on peut parler de carte cognitive. Huff opère un regroupement et confère aux cartes cognitives cinq buts que l'on pourrait placer sur un continuum allant de la «simple» analyse de contenu (l'expression verbale étant vue comme le reflet direct de l'activité mentale) à la modélisation d'une partie de la cognition (la carte doit alors faire l'objet d'analyses plus approfondies pour mettre en exergue la structure cognitive de l'individu, le chercheur devant alors s'astreindre à un travail d'interprétation plus important, car cette structure n'est pas entièrement accessible consciemment par le sujet). Ce regroupement fait apparaître cinq familles de cartes, dont les cartes causales que nous mobilisons. Une autre typologie consiste à distinguer les types de cartes suivants. Une carte cognitive correspond à une carte d'un individu (niveau idiosyncrasique), mais les auteurs ont tendance à utiliser le terme également pour le niveau collectif. Une étude au niveau organisationnel (niveau collectif) donne lieu à la construction d'une carte stratégique ( $c f$. les travaux de l'équipe d'Éden) issue de négociations entre les acteurs, des jeux 
de pouvoir et d'autres contingences émergeant de la structure «cognitive organisationnelle » et la contraignant, ce qui, dans le fond, correspond bien à leur réalité quotidienne. Quant aux cartes composites, elles se construisent par superposition de cartes individuelles de manière à juxtaposer les nœuds, liens et boucles. Chaque acteur apporte sa contribution à la carte composite dessinant le système social (Bougon et Komocar, 1994). Notre étude étant centrée sur le créateur d'entreprise, c'est aux cartes cognitives que nous nous intéressons et plus précisément aux cartes cognitives idiosyncrasiques causales ${ }^{4}$.

\subsection{Fondement de l'utilisation des cartes cognitives idiosyncrasiques causales}

Au sein de l'univers cognitif de l'individu se dessine une structure liant entre eux des facteurs, liens généralement causaux. D'autres types de liens existent (Axelrod, 1976; Huff, Nappareddy et Fletcher, 1990), mais il a été démontré par les psychologues et les psychologues sociaux qu'il existe au sein de l'univers cognitif une structure causale et que l'interprétation donne naissance à des représentations que se fait l'individu du monde sous forme de structures causales. Le schéma causal est, selon Kelley (1967), une conception générale que la personne a concernant la manière dont certains types de causes interagissent pour produire un type d'effets particuliers, sous l'influence notamment de ses expériences passées (mais aussi de ses émotions, Demasio, 1995 ; Vernier et Vincent, 1996). Pour Weick, les schèmes façonnent la réalité et sont façonnés par elle également si la rationalité limitée des individus les conduit à faire preuve d'une certaine «économie psychologique », cherchant plutôt à confirmer leurs schèmes actuels qu'à en élaborer de nouveaux, et cela d'autant plus que l'ambiguïté du monde et sa complexité sont telles qu'elles autorisent l'individu à interpréter des événements selon ses schèmes du moment (Cossette, 1994b). On rejoint en partie la théorie de l'équilibre cognitif (Heider, 1958) selon laquelle une contradiction entre les éléments de l'univers cognitif tente d'être résolue par la modification des rapports entre ces éléments ou par la modification de la représentation. La théorie de l'attribution de Heider, processus par lequel «l'homme appréhende la réalité et peut la prédire et la maîtriser », répond à ce besoin d'équilibre. $\mathrm{La}$ question reste posée à savoir si elle « renvoie à un processus d'analyse causale ou si les processus d'attribution reposent sur des structures de connaissance en fonction desquelles l'information est traitée » (Beauvois et Deschamps, 1990, p. 71). Ces visions ne sont pas antagoniques. Dans le premier cas, l'attribution est un processus de reproduction de la réalité où l'information subit un traitement

4. Pour connaître nos réserves sur l'utilisation des cartes collectives voir Verstraete $(1995,1996)$ 
statistique; dans le second, elle est un processus de construction de la réalité sociale où l'information serait « une matière première à travailler ; elle est sans cesse travaillée, remodelée, entre autres à l'aide de ces processus d'attribution » (Ibid.). Fondamentalement, l'acception heiderienne de l'attribution sous-entend la recherche par l'individu des causes d'un événement ; recherche influencée par l'évitement du déséquilibre cognitif, donc par une tendance à conférer à l'environnement une stabilité dans une logique de «cohérence cognitive ». En ce sens, l'attribution correspond bien à un processus de production de la réalité, avec une tendance à la rationalisation. On entre ici dans le champ paradigmatique de la « dissonance cognitive » (Festinger, 1957), chère à la psychologie sociale, à relier à la théorie de l'attribution, de l'équilibre et quelques autres qui, selon Beauvois et Deschamps (1990),

- portent sur l'univers cognitif des individus, sur un ensemble de savoirs, de connaissances et de croyances, ensemble appelé «cognition » (Festinger, 1957) ou encore « représentations cognitives » (Abelson et Rosenberg, 1958);

- définissent l'organisation particulière la plus satisfaisante, la plus harmonieuse des éléments de cet univers cognitif conférant au système ce que l'on appelle la consistance ; le travail cognitif consiste alors à rétablir ou à engendrer des états harmonieux.

Enfin, rappelons que pour Piaget (1967) le développement individuel comporte deux phases : une phase d'accommodation des schèmes d'assimilation à la réalité (perçue) présente et une phase d'assimilation de la réalité, correspondant à une perception qui permet à l'individu, au regard de sa structure mentale, d'appréhender cette réalité.

Nous nous intéressons dans cet article aux cartes reflétant justement la structure causale de la représentation. Comme nous l'avons souligné, d'autres liens unissent les éléments des schémas d'interprétation. Ainsi pour Hume $(1983)^{5}$, il existe, en plus de la causalité, l'analogie (les événements se ressemblent) et la contiguïté (les événements se sont produits en même temps). Pour Weick et Bougon (1986), les événements comportent quatre possibilités constituant les primitives de toute organisation : elles résultent d'événements similaires ou différents survenant au même moment ou à des moments différents. La combinaison de différents événements à différents moments engendre les inférences de la causalité.

5. Hume, D. (1983), Enquête sur l'entendement humain, Paris, Flammarion, réédition, (éd. or. 1758). 
Tableau 1

Les primitives épistémologiques

\begin{tabular}{lccc}
\hline & Moments auxquels se produit l'événement & \\
\hline & & Identiques & Différents \\
Classification & Identiques & $\mathrm{A}$ & $\mathrm{B}$ \\
des événements & & Identité & Sériel \\
& Différents & $\mathrm{C}$ & $\mathrm{D}$ \\
& & Corrélation & Causalité \\
\hline
\end{tabular}

Source: Weick et Bougon (1986), «Organizations as cognitive maps: charting ways to success and failure X, dans Sims et Giois (éd.), The Thinking Organization, San Francisco, Jossey-Bass, p. 102-135.

Dans la cellule A du tableau précédent, les mêmes événements se produisent en même temps, cette combinaison d'événements peut être appelée «coïncidence» ou «identité». La cellule $B$ représente le cas où les mêmes événements se produisent à des moments différents, et peut être qualifiée de «sériel ». En C, des événements différents se produisent en même temps; les auteurs parlent de corrélation. Enfin, la cellule $\mathrm{D}$, où différents événements se produisent à des moments différents, renvoie à la causalité. Un individu ayant observé deux événements différents à des moments différents est susceptible d'établir un lien de causalité entre eux, lien pouvant être spéculatif et arbitraire. Weick et Bougon se concentrent, dans leur article, sur les cartes cognitives causales que les individus construisent de leur univers organisationnel, l'organisation étant vue comme un instrument permettant de faire des choses, comme un outil consciemment construit, comme une institutionnalisation des relations «moyens / fins» et des assertions «si / alors».

\subsection{Objet et principe de la cartographie cognitive causale}

La cartographie cognitive consiste «à représenter les processus de pensée des décideurs comme orientés par des agencements d'items reliés entre eux par des relations » (Laroche et Nioche, 1994, p. 67). Elle est « une représentation graphique de la représentation mentale que le chercheur se fait d'un ensemble de représentations discursives énoncées par un sujet à partir de ses propres représentations cognitives à propos d'un objet particulier » (Cossette et Audet, 1994, p. 15). Cette définition, que nous retenons, appelle quelques commentaires. Elle permet en premier lieu d'insister sur le fait que la cartographie cognitive n'a pas l'ambition d'obtenir la cognition complète d'un individu, mais une partie de sa cognition : celle relative à un objet particulier; en second lieu, qu'il s'agit de la représentation (celle du chercheur) d'une représentation (celle du sujet). Le danger, et c'est ce qui expose la cartographie à la critique péjorative, réside 
dans l'utilisation, par exemple, de cet outil après des d'entretiens non enregistrés ; le chercheur accède alors plus à ses propres schèmes d'interprétation qu'à ceux du sujet. La «mode», justifiée, de la cartographie conduit inévitablement à ce type d'excès. Il appartient au méthodologue de faire le tri. Sans prétendre à une modélisation, la figure suivante simplifie la description du processus.

FIGURE 1

La cartographie cognitive : une représentation d'une représentation

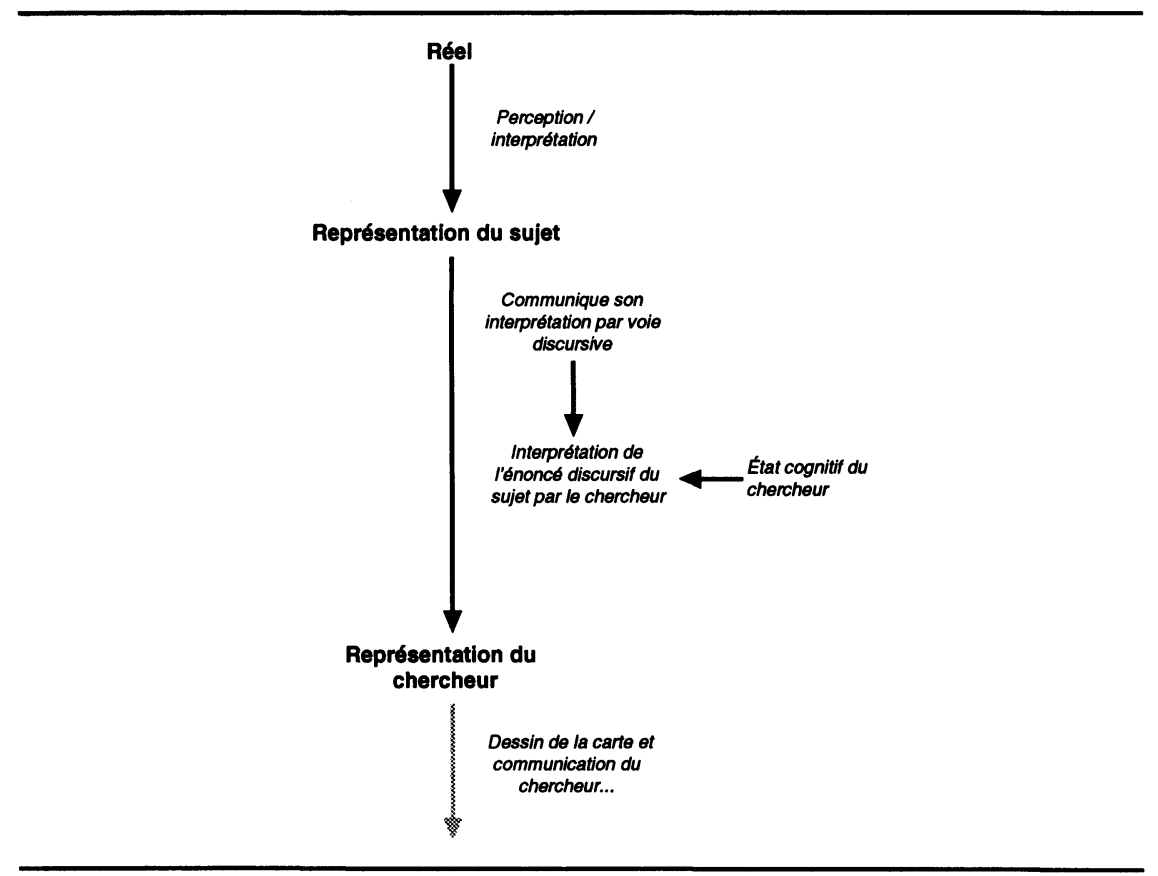

Le sujet perçoit, interprète le réel et communique la représentation qu'il s'en fait par voie discursive au chercheur. Ce dernier va interpréter l'énoncé discursif de la représentation du sujet pour se faire sa propre représentation de la représentation du sujet... Notons que l'état cognitif du chercheur n'est pas neutre en ce qui a trait à la façon dont il va interpréter l'énoncé discursif, dont il va construire sa propre représentation. Éventuellement, le chercheur peut à son tour communiquer sa représentation de la représentation du sujet à un acteur qui lui-même se fera une interprétation... Le chercheur a donc sa propre représentation de la représentation du sujet. Il ne peut jamais être totalement neutre. Il est clair que les adeptes popperiens trouveraient difficilement dans ces approches à appliquer le critère de réfutabilité. Dans une discipline comme la psychologie sociale, les connaissances liées à la logique formelle 
sont opposées à celles liées à la logique naturelle ( $c f$. Grize, 1989). La première, celle de la démonstration mathématique, ne dépend ni des sujets qui la produisent ni des circonstances de sa production ; la seconde, celle de la pensée sociale, est déterminée par le contexte dans lequel elle s'inscrit.

[...] en d'autres termes, on oppose le sujet optimal qui fabrique des démonstrations totalement dépendantes de normes prétablies et particulièrement invariantes, au sujet social qui, lui, a pour caractéristique essentielle d'agir et de penser en interaction. Or, on peut penser que les représentations sociales constituent un cas particulier de la connaissance ou, si l'on préfère, qu'elles appartiennent à la classe des connaissances principalement déterminées par la logique naturelle.» (Guimelli, 1994, p. 179)

Le critère de réfutabilité est en fait le propre de la pensée humaine. On a vu que l'individu fonctionne «à l'économie » préférant réfuter et minimiser la portée des informations nouvelles (Moliner, 1994) plutôt que de prendre le risque d'un éclatement de la représentation qu'il a. «En ce sens, le processus de réfutation est probablement un puissant agent de stabilité des représentations sociales.» (Ibid., p. 220) Cette apparente limitation ne doit pas rendre les utilisateurs potentiels de la cartographie cognitive timides à son égard, et cela pour au moins deux raisons. La première est que, dans notre quotidien, ces processus sont sans cesse à l'œuvre. La seconde réside dans la méthode déployée. À ce titre, celle que nous proposons ici mobilise deux acteurs : le chercheur et le sujet individu; et le caractère circulaire des échanges tend à mettre en phase leur interprétation respective au regard de l'objet de leurs rencontres, une phase de validation réduisant le risque de déphasage d'interprétation.

L'intention de la cartographie cognitive «est de décrire une perception consciente de la réalité avec suffisamment de détails pour capturer la perception idiosyncrasique qu'a du monde un individu » (Langfield-Smith, 1992, p. 350), sans chercher une description exhaustive de ses croyances, mais de présenter un «modèle simulant sa cognition actuelle sur un domaine précis » (Ibid.). Le chercheur dessine la carte cognitive à partir du discours, oral ou écrit, du sujet en reliant les concepts énoncés, par exemple, par des liens de causalité pour ce qui nous intéresse. Le discours étant une production du sujet, les approches de type mécaniste d'obtention d'informations sont à proscrire (Cossette et Audet, 1994).

Les concepts qu'une personne utilise sont représentés par des points, et les liens de causalité entre ces concepts sont représentés par des flèches qui les unissent (Axelrod, 1976). Leur forme graphique permet de voir assez facilement comment les concepts et les relations causales s'agencent.

Dans une carte causale, les concepts sont reliés en termes de cause / effet même si parfois le sujet ne sait pas donner le sens de l'influence. En fait, comme le souligne Cossette, l'influence peut être possible ou réelle, le rapport 
peut être du type moyen / fin ${ }^{6}$, cause / effet et la variable initiale peut être une condition à l'existence de la variable finale. La distinction entre un lien de type moyen / fin et cause / effet est d'importance puisque «dans une relation de cause à effet, l'explication d'un événement se trouve dans ce qui le précède, dans ses antécédents, alors que dans une relation de moyen à fin, elle se trouve dans ce qui suit l'événement, dans ses conséquences" (Cossette, 1994b, p. 146). Pourtant, il est parfois difficile de dire de quel type de relation relève le lien. Ainsi en est-il de celui unissant les variables «rentabilité » et «croissance de l'entreprise». Dans l'exemple suivant, emprunté à Cossette, autant le volume des ventes $(\mathrm{X})$ pourrait conduire à la survie de l'entreprise $(\mathrm{Y})$ dans la mesure où celle-ci est rentable $(\mathrm{A})$, autant cette rentabilité ne pourrait exister que dans la mesure où le volume des ventes $(\mathrm{X})$ conduit à une baisse des coûts fixes $(\mathrm{Z})$ :

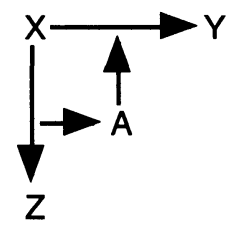

Les cartes sont dessinées à partir de l'énoncé discursif du sujet, énoncé conceptualisant les représentations cognitives du sujet. Lors de cette opération de schématisation, les représentations cognitives sont à la fois conservées, transformées et réduites (Cossette et Audet, 1994). Une schématisation est la mise en discours du point de vue qu'un locuteur A (lorsqu'il parle, A traduit la façon dont il voit le monde, mais qu'il se donne encore à voir lui-même) se fait d'une certaine réalité $R$ (une schématisation est un objet sémiotique). Cette mise en discours est faite pour un interlocuteur ou un groupe d'interlocuteurs $B$ dans une situation d'interlocution donnée... (Grize, 1989). Les sujets s'expriment dans une langue naturelle, dont le sens ne peut pas être forcément immédiatement décodé soit parce que l'interlocuteur n'était pas le destinataire de la communication, que la citation est détachée de son contexte, ou encore parce qu'il convient de se référer à des «préconstruits » culturels nécessaires à la compréhension de la citation. Sur ce dernier point, Grize prend comme exemple la citation suivante: «je n'ai quand même pas la tête dans le sable» dont la compréhension oblige de savoir ce que le locuteur prête aux comportements des autruches. La mise en discours est faite pour un interlocuteur, ou un groupe d'interlocuteurs B : «l'image qu' une schématisation offre de ce dont elle traite est fonction de celui, ou de ceux, au(x)quel(s) elle est destinée. Elle n'est pas authentiquement celle de $\mathrm{A}$. Remarquons d'ailleurs qu'il ne s'agit pas là d'une question de sincérité, mais d'une adaptation nécessaire à ceux dont on veut être

6. La variable finale (fin) peut être un but ou un non-but, c'est-à-dire un résultat à éviter. 
entendu » (Grize, 1989, p. 157). Le terme « authentiquement » est important. On ne peut pas accéder directement à la cognition d'un individu, lire dans son cerveau comme on lit dans un livre. L'accès à la cognition de l'individu se fait à travers ce que dit le sujet. À ce titre, le discours n'est pas neutre d'intention : on peut lui conférer, comme à toute communication, l'ambition d'atteindre un but. L'adaptation dont parle Grize se fera conformément aux ambitions du locuteur.

Ces développements, démonstratifs de notre conscience des limites de l'outil, soulignent implicitement le caractère instrumental de la cartographie cognitive : « [...] nous ne pouvons présumer que la carte cognitive, un produit matériel, soit la copie parfaite du produit cognitif que le chercheur a dans la tête, ni que cette représentation mentale du chercheur soit le reflet fidèle de représentations discursives du sujet, ni que celles-ci soient équivalentes aux représentations cognitives de ce dernier. Et nous ne pouvons encore moins supposer que ces représentations cognitives, de nature sémiotique, correspondent à la réalité telle qu'elle est vraiment. » (Cossette et Audet, 1994, p. 33) Toutefois son caractère « émancipatoire » mérite d'être reconnu plus largement (Audet, 1994). La capacité réflexive de l'individu détermine le niveau potentiel d'utilisation du caractère émancipatoire de sa carte. Si elle est forte, il a tout intérêt à y avoir accès, cela l'aidera dans ses interactions multiples.

\subsection{Complexité d'une carte, centralité des concepts}

Une carte causale contient un nombre variable de concepts selon la durée de l'entretien, la compétence de l'interviewer, l'ouverture des questions et le type de carte. La relative complexité d'une carte s'exprime notamment :

- par le nombre de concepts ;

- par la « centralité » de certains d'entre eux (un concept est généralement dit central lorsque de nombreux liens en partent ou y arrivent);

- par le nombre de liens ainsi que par le nombre de concepts influencés (ce qui est source de situations conflictuelles);

- par l'interconnexion des concepts : une carte peut se placer sur un continuum allant de la carte où aucun des concepts n'est relié à un autre, à la carte où tous les concepts sont reliés entre eux. Ces deux cas extrêmes sont peu probables, surtout le premier. Néanmoins, il peut arriver qu'une carte fasse apparaître des îlots, des ensembles de concepts isolés les uns des autres, l'ensemble de la carte s'apparentant à un assemblage de petites cartes, témoin d'une complexité moins grande, d'une simplification de la réalité faite par le sujet ( $c f$. processus de catégorisation).

Un nombre important de facteurs «influençants » est une indication de multiplicité d'options possibles pour résoudre le problème. Le calcul de ratios, 
par exemple le nombre de concepts influençants sur le total des concepts, peut être une méthode de mesure de la complexité (un logiciel comme Graphics Cope effectue ce type de calcul).

En s'inspirant des travaux sociologiques, les concepts les plus centraux peuvent être identifiés de plusieurs façons (Lazega, 1994) ${ }^{7}$ :

- la centralité de type «degree » se mesure au nombre de liens établis entre un concept et les autres, plus un concept est central plus il est actif dans le réseau ;

- la centralité de type «closeness » se mesure au «nombre de pas » que doit faire un concept pour rejoindre les autres membres du réseau; la centralité renvoie à la proximité, un concept central peut rentrer vite en contact avec les autres ;

- la centralité de type «betweeness » se mesure au nombre de chemins les plus courts sur lesquels le concept est un passage obligé entre deux autres concepts, un tel concept central contrôle les interactions entre d'autres acteurs.

La mesure de ces centralités renvoie à l'utilisation d'algorithmes issus de la théorie des graphes, ce qui présente des limites en cartographie cognitive (idiosyncrasique) qui tiennent aux points suivants :

- la théorie s'inscrit généralement dans l'espace métrique euclidien, et les modèles, pour lesquels l'espace est considéré comme le support des interrelations entre groupes d'individus, font largement appel à la notion de distance ;

- la liste des variables présentes sur une carte cognitive n'est pas exhaustive. Il est possible de s'appuyer sur le principe d'agrégation / désagrégation pour le prouver. En effet, nous avons pu constater qu'il suffit de s'attarder davantage sur un concept lors de l'entretien pour qu'aussitôt de nouveaux éléments soient énoncés. Cela peut amener le chercheur «à découvrir ce qu'il cherche ». Certes, il est toujours possible d'évoquer le principe de saturation, mais il peut être, nous semble-t-il, manipulé, consciemment ou inconsciemment par le sujet ou le chercheur, ou les deux à la fois ;

- les variables évoquées par les sujets lors de la collecte des données sont subjectives et il est en partie contradictoire de vouloir formaliser les relations entre données subjectives par nature;

7. Le concept de centralité est utilisé également en psychologie sociale. Voir à ce propos le texte de Moliner (1994). 
- l'univers cognitif du sujet est transitoire. À ce titre, formaliser les relations existantes entre concepts relève d'un faible intérêt dans le cadre d'une carte idiosyncrasique.

Ces restrictions sont relatives au traitement des cartes cognitives idiosyncrasiques pour relever l'importance des concepts selon leur centralité et selon des méthodes utilisant les algorithmes de la théorie des graphes. Cela ne veut pas dire que ces traitements soient à exclure, car ils permettent de mettre en relief certaines caractéristiques intrinsèques des cartes. Mais ils doivent être pondérés par une interrogation directe du sujet sur ce qu'il considère comme le plus important dans sa carte.

Il en est autrement pour ces restrictions en ce qui concerne les cartes stratégiques et les cartes composites, et l'on peut alors se rapprocher de l'analyse structurale utilisée dans le cadre de l'étude des réseaux sociaux, type d'analyse que Degenne et Forsé (1994) classent comme paradigmatique. Ce type d'algorithmes est également utilisé dans le cadre des études prospectives (sur la prospective, voir Baumard, 1996 ; Buigues, 1985 ; Godet, 1991a, 1991b ; Hatem, Cazes et Roubelat, 1993). Nous avons, à ce titre, proposé la cartographie cognitive comme outil de collecte de données permettant d'alimenter une base (tout comme la méthode Delphi ou la mini-Delphi) susceptible de subir ce type de traitement, base utile à la prospective (internationale, nationale, sectorielle ou organisationnelle) [Verstraete, 1996b].

\section{Cadre opératoire}

Rappelons que l'idée est d'appréhender l'univers cognitif du créateur relatif à son projet, d'identifier les facteurs qu'il juge les plus importants pour sa réussite (FCS et FSR), de relever la façon dont il maîtrise ces facteurs puis de l'aider dans la mise en place d'un contrôle (selon l'acception large du terme) après l'avoir aidé à mettre au point sa vision. Pour cela, nous mobilisons la cartographie cognitive.

Notre technique de recueil de matériaux s'inspire directement d'une proposition faite par Pierre Cossette (1994c) (dont la préoccupation était de fournir une méthode systématique d'aide à la mise au point de la vision des dirigeants) qui se décompose en trois phases séquentielles. Respectueux de la méthode initiale pour les deux premières phases, nous avons adapté la suite à notre étude. Cinq phases composent notre démarche: une phase d'exploration, une phase de validation, une phase d'analyse (ou de tri), une phase d'interrogation (ou de sensibilisation) et une phase de décision. Chacune de ces phases sera analysée dans les sous-sections présentées ci-après. L'expérience a été menée dans une entreprise de distribution de produits bien spécifiques qui accueillait le public depuis un trimestre dans sa surface commerciale. Nous appellerons son créateur M. Petit. 


\subsection{La phase d'exploration}

Cette phase débute par une question ouverte afin de s'inscrire effectivement dans le référentiel de l'individu. L'interviewé est invité à relever les variables qui, selon lui, ont un impact sur l'avenir de son entreprise. Chacune de ces variables est placée par l'accompagnateur au centre d'une «grille d'exploration systématique » (figure 2).

\section{FIGURE 2}

\section{Grille d'exploration systématique}

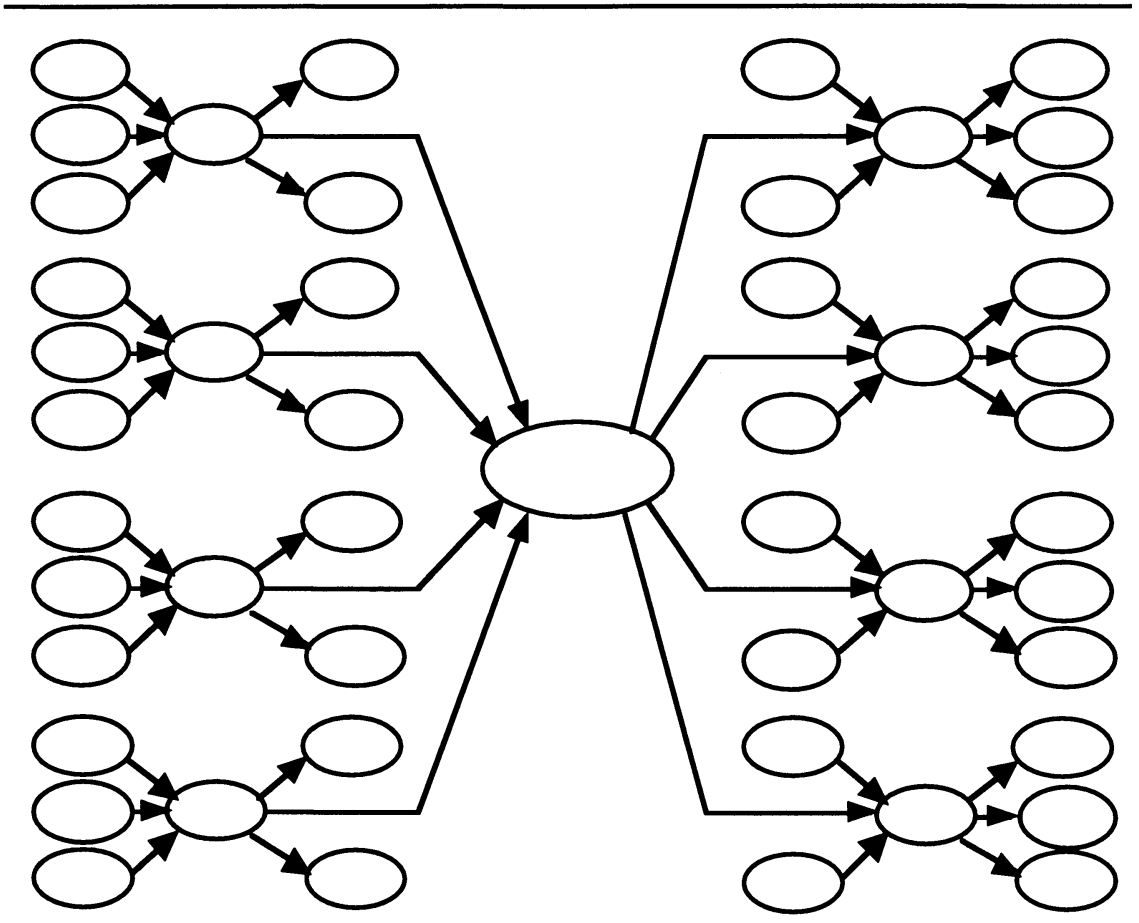

Source :Cossette, P. (1994c), « Développement d'une méthode systématique d'aide à la mise au point de la vision stratégique chez le propriétaire dirigeant de PME : une étude exploratoire », Colloque PME de Strasbourg, p. 73-82.

La suite de cette phase d'exploration consiste à revenir sur chacune des grilles, à demander au sujet quelles variables ont une influence sur celle du centre et les variables que cette dernière influence. L'opération est réitérée pour les «explications » (variables influençantes) et les «conséquences» (variables influencées). Deux remarques s'imposent ici : il est possible qu'un même facteur se retrouve sur plusieurs grilles et il n'est pas obligatoire de remplir tous les 
espaces prévus. Une carte causale est dessinée à partir des matériaux recueillis. Il s'agit en fait de regrouper sur un même support les différentes grilles.

L'intérêt principal de la présentation de la grille d'exploration systématique vierge réside dans la sensibilisation du sujet aux attentes de l'accompagnateur. Une démarche d'entretien classique avec enregistrement peut donner lieu à la construction d'une carte, mais l'un des avantages de la méthode proposée par Cossette est son caractère explicite et systématique, caractéristique réclamée par Huberman et Miles (1991, p. 24) : «le champ de la recherche qualitative a désespérément besoin de méthodes explicites et systématiques pour établir les conclusions et les tester rigoureusement, méthodes pouvant être réutilisées par d'autres chercheurs, exactement comme les tests de signification et de corrélations le sont par les chercheurs quantitatifs».

\subsection{La phase de validation}

Pour tester sa validité, la carte tracée à l'aide des grilles d'exploration systématique est présentée au sujet pour amendement éventuel (trois jours plus tard dans le cas de M. Petit). Cette phase consiste à expliciter verbalement au sujet la façon dont son énoncé discursif a été représenté graphiquement. Est ainsi vérifiée l'interprétation partagée, entre l'accompagnateur et l'accompagné, de chacun des concepts (ce qui permet d'avoir sur la carte des concepts relativement simples dans leur expression, un simple mot suffisant parfois) et l'existence d'un lien entre deux concepts. Après chaque explicitation d'un triplet concept-lien-concept (qui conduit parfois à expliciter une chaîne plus longue, voire une boucle complète), la validation s'exprime par amendement éventuel ou par acquiescement direct du sujet. Les concepts et le lien les unissant sont alors surlignés au crayon fluorescent par le sujet et le chercheur, tous les deux étant équipés d'une carte d'une taille respectable (format A3) et d'un crayon. L'avantage de cette démarche est qu'elle permet au sujet d'appréhender totalement la représentation graphique, ce qu'il fait sans mal puisqu'elle reflète son énoncé discursif et, aussi fidèlement que possible ( $c f$. Grize), une partie de sa cognition.

TABLEAU 2

Types de lien et leur représentation graphique

\begin{tabular}{lll}
\hline Type de lien & Représentation graphique \\
\hline A influence B & A & $B$ \\
\hline une augmentation de A entraîne une diminution de B & $A$ & $B$ \\
\hline A est une composante de B & $A$ & $B$ \\
\hline
\end{tabular}


FIGURE 3

Carte cognitive de M. Petit tracée en juin 1995

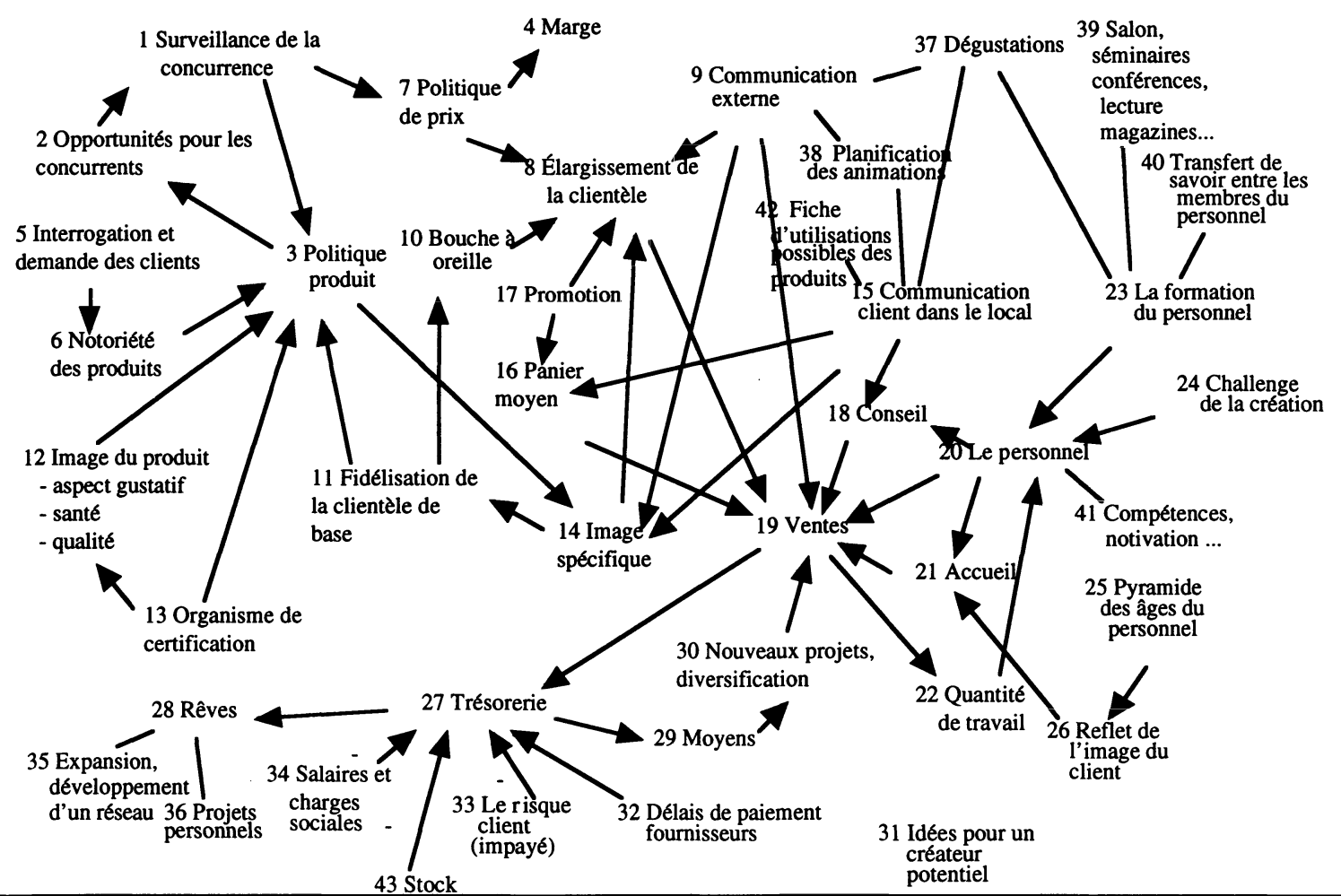




\subsection{La phase d'analyse ou de tri}

Pour l'analyse de la grille validée, Cossette a utilisé un logiciel dédié. Cette analyse porte, entre autres, sur l'importance des concepts, leur similitude (regroupement), les facteurs influençants, les facteurs influencés et les éventuelles boucles dans la carte (A influe sur B qui influe sur C qui influe sur A). S'agissant du calcul du nombre de liens unissant un concept à d'autres et du repérage des boucles, ce type de logiciel permet une économie certaine au chercheur. S'agissant de mesurer l'importance d'un concept, nous renvoyons le lecteur aux réserves que nous avons plus généralement formulées relativement à l'utilisation de certains algorithmes dans le cadre d'une analyse d'une carte idiosyncrasique. Ainsi, M. Petit considère le concept «fidélisation de la clientèle », qui n'est relié qu'à trois autres concepts, aussi important que le concept «ventes», relié celui-là à neuf autres concepts (les facteurs retenus furent les suivants : les ventes, 15 points ; la fidélisation de la clientèle de base, 15 points ; l'accueil, 10 points ; l'élargissement de la clientèle, 10 points ; la marge, 10 points ; la trésorerie, 10 points ; la politique produit 6 points ; la qualité des produits, 6 points ; la politique de prix, 6 points ; le bouche à oreille, 6 points ; l'image spécifique, 6 points). La «marge » est également perçue comme FCS, mais n'est reliée qu'à un seul autre concept. Remarquons qu'elle n'est reliée ni aux ventes ni à la trésorerie, et cela s'explique. Le présent texte ayant une visée opérationnelle et la place qui nous est réservée ayant ses limites, nous ne commenterons pas la vision stratégique de $\mathrm{M}$. Petit ${ }^{8}$. Nous avons maintenu le terme d'analyse pour cette phase, car les matériaux recueillis par un chercheur qui déploierait les deux premières phases peuvent donner lieu à d'intéressantes analyses, ce que nous faisons dans le cadre d'une autre étude.

Les concepts sont ensuite présentés sous forme de liste au sujet et il lui est demandé de faire ressortir ceux qu'il considère comme les plus importants (la proximité de certains concepts le conduit à opérer parfois des regroupements ou à choisir un facteur générique) et de pondérer ceux-ci par distribution de cent points entre les facteurs retenus. Il lui est précisé qu'à un extrême, il peut attribuer les cent points à un seul concept, à un autre extrême, donner quelques points à tous les concepts. Cette étape de classement et de pondération oblige le sujet à se centrer sur la question des facteurs importants et peut donner lieu à des analyses. Des techniques proches sont utilisées en psychologie sociale. Par exemple, Abric (1994), après le repérage des liens entre les éléments de la représentation, propose de hiérarchiser les sujets les plus fréquemment produits, puis de les présenter au sujet sous forme de fiches. Celui-ci doit séparer les

8. Le lecteur peut s'adresser à l'auteur pour recevoir un texte narratif permettant d'appréhender la carte présentée. 
fiches en deux paquets, en mettant dans l'un les sujets les plus caractéristiques de l'objet étudié, et dans l'autre, les moins caractéristiques; l'opération est réitérée pour le paquet constitué des sujets les plus caractéristiques, etc. Cette méthode permet d'obtenir un classement par ordre d'importance et a été validée expérimentalement pour repérer la centralité de certains éléments dans une étude portant sur la représentation de l'artisan (Abric, 1984, 1989). Cette technique des tris hiérarchiques successifs connaît des variantes. Au regard de notre objet d'étude, nous avons préféré que le sujet affecte lui-même le poids respectif des facteurs, et plutôt que d'imposer au sujet la technique à utiliser pour hiérarchiser les sujets, nous le laissons opérer selon la technique qu'il souhaite. Il peut ainsi commencer par cocher les éléments qu'il retient (il leur affecte les points plus tard) ou, par barrer ceux qu'il ne retient pas ou par affecter directement les points, etc. Laisser l'individu libre de la technique de hiérarchisation nous semble plus proche de ce qu'il fait habituellement dans une situation de choix. Il en est de même pour l'affectation des scores, puisque l'individu peut exprimer librement le poids qu'il attribue à tel ou tel sujet, ce qui est mieux si l'on veut connaître la « représentation managériale », d'autant plus que notre travail se situe sur le plan idiosyncrasique.

\subsection{La phase d'interrogation ou de sensibilisation}

Pour chacun des facteurs que le créateur a mis en exergue lors de la phase précédente, on lui demande comment il le contrôle ou a l'intention de le contrôler. Il s'agit donc d'interroger explicitement le créateur relativement aux procédures, formelles ou informelles, qu'il utilise pour suivre, voire maitriser, l'évolution des facteurs. Implicitement, c'est la sensibilisation au besoin de contrôle que provoque cette interrogation. En effet, le créateur se rend compte éventuellement, et ce fut le cas lors de notre expérience, que tel ou tel facteur, qu'il considère comme très important pour la réussite de son entreprise, ne fait l'objet d'aucun suivi et que rien n'est fait pour s'assurer de sa maîtrise ou de prévoir des parades lorsque le facteur est plutôt un FSR (notons qu'un apparent FCS peut être un FSR parce que le créateur n'a pas les ressources nécessaires permettant sa maîtrise). Cela vaut tous les discours d'experts du monde, car cette prise de conscience, le créateur l'opère dans son propre référentiel, il n'y est que d'autant plus sensible. Ainsi, l'un des soucis de M. Petit est de pouvoir rapidement faire le point sur sa situation financière. Mis à part l'état de rapprochement bancaire qu'il effectuait à chaque réception d'un relevé de banque, aucun dispositif ne permettait de savoir «si cela passe ou si cela casse » pour reprendre l'une de ses expressions. Pourtant, tous les outils étaient disponibles, mais, par exemple, l'investissement informatique initial était utilisé uniquement pour gérer les fiches produits, éditer les étiquettes code barre et les bons de commande, et connaître les statistiques de ventes. La notion de besoin en fonds de roulement, bien connue du gestionnaire, 
ne l'est pas forcément du créateur. C'est d'ailleurs une constatation malheureuse régulièrement pointée comme cause de défaillance (une bonne appréhension de cette notion devant conduire à une juste mesure des ressources nécessaires à la croissance). L'exemple paraît trivial, mais constaté chez M. Petit. Ce constat est d'autant plus intéressant qu'il avait suivi une formation en gestion. En fait, si M. Petit connaissait l'importance de la notion de besoin en fonds de roulement (BFR), soit il avait des lacunes pour la mise en place de la procédure permettant de le mesurer (ou du moins d'en avoir une appréciation correcte) parce qu'on ne lui avait pas expliqué la démarche pragmatique afférente, soit cette mise en place ne lui apparaissait pas comme une priorité ; il nous semble qu'il y avait un peu des deux.

Cet exemple renvoie à une vision comptable du contrôle, mais l'expérience a été plus loin et a permis d'étudier les possibilités de contrôle dans une acception large du terme. En effet, à travers les discussions qu'a provoqué le caractère «émancipatoire » de la carte, les aspects stratégiques ont été largement abordés. Un avantage de la représentation graphique est que le sujet « voit » sur quoi il faut agir (la maîtrise d'un facteur peut être induite par les facteurs qui l'influencent), «voit les alternatives», etc. (cf. Audet, 1994). C'est lors de la phase de décision que ces aspects se sont exprimés. Un point important doit auparavant être souligné (même si l'expert en a sans doute conscience, des rappels évitent de tomber dans certains biais) concernant l'approche par les FCS. Elle ne doit pas conduire à prôner la dominance d'une ou de quelques variables, le système est à «multiples entrées, c'est-à-dire que l'on peut obtenir un effet en agissant sur une variable qui n'est ni la plus profonde ni la plus décisive» (Crozier et Friedberg, 1977, p. 380). Les caractéristiques du système sont à étudier de plus près.

\subsection{La phase de décision}

C'est l'aboutissement de la réflexion qu'a amorcée la phase précédente. La carte joue ici pleinement son rôle « émancipatoire » et les connaissances de l'accompagnateur sont en mesure de s'exprimer; d'une part, parce que cet accompagnateur s'est inscrit dans le référentiel du sujet (il retire donc, par rapport à sa tâche, autant de bénéfices que le créateur de la méthode), d'autre part, parce que ce dernier, grâce à la phase de sensibilisation, est réceptif au discours qui lui est tenu. L'accompagnateur apporte ses connaissances à la fois pour la mise en place des outils nécessaires à la gestion de l'entreprise (selon, bien évidemment, les possibilités de la firme) et pour la mise au point de la vision de l'accompagné et le repérage des possibilités de changements stratégiques. Les deux aspects sont intimement liés, car on ne saurait mettre en place un contrôle, qu'il soit stratégique ou opérationnel, sans considérer ce qui est retenu comme facteurs déterminants pour la réussite de l'entreprise (Anthony, 1965 ; Ardoin, Michel et 
Schmidt, 1986 ; Bouquin, 1986). Nous renvoyons ainsi au principe de déclinaison de la stratégie, principe bien connu des contrôleurs. Ceux-ci pourront d'ailleurs, nous semble-t-il, tenir compte de la présente proposition d'utilisation de la cartographie cognitive pour la mise en place de système et procédures de contrôle dans les entreprises naissantes, dans les TPE ou PME, le préalable étant peutêtre de scruter la vision stratégique du dirigeant.

Cette phase a été appelée " phase de décision », car elle doit aboutir, selon justement le principe de déclinaison de la stratégie, à des décisions d'actions autant d'ordre stratégique qu'opérationnel, au moins à des intentions ou à des définitions d'actions si l'accompagnateur ne reçoit pas la mission de la mise en œuvre. Dans le cas contraire, une phase d'action est ajoutée à la méthode.

Après la phase de décision, un récapitulatif doit être établi ; un tableau de bord, outil privilégié pour le suivi des FCS, est proposé.

\section{Discussion}

Deux séances ont été nécessaires. La première fut réservée à l'exploration et les autres phases se sont enchaînées lors d'un même après-midi, trois jours plus tard. La phase d'exploration a pris deux bonnes heures, la phase de validation moins d'une heure et la phase de tri, une vingtaine de minutes. La phase d'interrogation (ou de sensibilisation) a demandé une heure et demie (la liste des concepts étant amendée de façon concomitante à l'amendement de la carte), la phase de décision environ deux heures. En ajoutant aux étapes sur le terrain le temps nécessaire au tracé de la carte, les temps de déplacement, de prise de rendez-vous et d'établissement du rapport final, il faut prévoir une journée et demie, voire deux journées d'accompagnateur. Cette information est d'importance pour les organismes susceptibles d'être intéressés. Rappelons qu'il semble, d'après les statistiques mesurant le taux de défaillance des jeunes entreprises, que les risques d'échec sont moindres en cas d'accompagnement. Soutenir l'entrepreneuriat est une chose ; son corollaire devrait être la pérennité de l'entreprise. La démarche proposée répond à cet enjeu. Elle ne défavorise pas (trop) les firmes plus anciennement installées, d'abord, parce qu'elle est ponctuelle, ensuite, parce qu'elle n'est pas financière, et enfin, parce que ces firmes peuvent elles-mêmes la solliciter pour un coût raisonnable.

Il est difficile d'estimer le temps nécessaire à une éventuelle phase d'action, c'est-à-dire la mise en œuvre du contrôle, car sa durée dépend de l'étendue de la mission et de la complexité du site investi. Lorsque l'organisation a atteint un certain niveau de complexité, une phase d'observation du terrain, durant laquelle le contrôleur ou l'auditeur déploiera les méthodes qu'il maîtrise, devient nécessaire. Il pourra alors vérifier la cohérence ou l'adéquation des systèmes et procédures de contrôle avec les attentes de la direction, d'une 
part, et considérer les remarques des opérationnels, d'autre part. Comme ces derniers sont en prise directe avec le marché, ils sont également porteurs, on le sait, d'informations stratégiques.

Nous nous permettons d'ajouter ici quelques remarques concernant les phases d'exploration et de validation.

- Il est profitable de rencontrer et de discuter avec les sujets avant l'expérience. Nous avions rencontré plusieurs fois M. Petit avant cette expérience. Les conditions expérimentales ont donc bénéficié d'un climat particulièrement propice minimisant, voire évitant, par là certains biais relatifs à la non-appréhension de la logique naturelle (cf. Grize, 1989) du sujet, d'une part, et à la méfiance dont il pourrait faire preuve, d'autre part. Il convient ici de préciser que $M$. Petit n'était pas informé des intentions relatives à la mise en place d'un contrôle. En fait, cette intention n'est même apparue que plus tard. En effet, le cas de M. Petit représentait initialement un terrain pour une recherche davantage théorique qu'opérationnelle, recherche pour laquelle les deux premières phases de la méthode proposée par Cossette (phase d'exploration et phase de validation) sont mobilisées, et au cours de laquelle il nous a semblé possible de développer une technique d'accompagnement concernant la mise en place d'un contrôle. La recherche «académique» a initié une recherche «pragmatique».

- Si possible, mener les entretiens en dehors du lieu de travail de l'intéressé ou faire en sorte que le sujet demande expressément qu'on ne le dérange pas. Cette précaution est surtout recommandée lorsque les sujets sont des créateurs d'entreprise ou des dirigeants de TPE. Ils sont généralement fortement impliqués dans les tâches de l'entreprise et ont beaucoup de mal à s'en détacher. C'est particulièrement vrai pour des techniciens, par exemple. Même s'ils accordent au chercheur le temps nécessaire à l'expérience, cette forte implication risque d'être source de nombreuses interruptions provoquées par les subordonnés, habitués à ce que tel ou tel problème, même mineur, soit abordé par le dirigeant. Il est alors difficile de mener cette expérience dans de bonnes conditions et cela nuit au caractère systématique de la méthode.

- Alterner, lors de la phase d'exploration, focalisation sur les grilles (lorsque le cheminement de pensée du sujet le permet) avec technique semi-directive tout en veillant à ce que celle-ci ne prenne le pas sur la technique initiale (ce qui nuirait au caractère systématique). Lors d'une expérience avec un autre créateur, ce dernier a évoqué au milieu de l'entretien un manque d'inspiration... il n'avait pas « la tête à cela »... Nous avions remarqué qu'il cherchait à remplir les cases de la grille d'exploration, sa réaction a été motivée par l'impression qu'il avait de 
ne pas être productif, les grilles n'étant certainement pas suffisamment noires à ses yeux. Il nous semble évident que si des relations n'avaient pas préexisté, cet entretien aurait été parasité de biais majeurs. Par exemple, le sujet aurait cherché à tout prix à « remplir». Hormis le fait que cela constitue en soi un biais, la validité de la carte (même si la seconde phase est prévue à cet effet, la construction initiale est d'importance) aurait été compromise, car le sujet aurait été amené à proposer des concepts et des liens ne constituant pas un bon reflet d'une partie de sa cognition. Nous avons introduit une démarche semi-directive pour que le sujet puisse se détacher des grilles et relancer ainsi l'expérience. Voyant qu'à travers ses propos nous remplissions les grilles, il s'y est tantôt replongé tantôt détaché, à sa convenance, et a été dès lors très à l'aise. Il nous semble clair que ce créateur avait vécu le début de l'entretien comme un exercice au sens scolaire du terme. Cet essai a évidemment eu de l'influence sur la façon dont nous avons mené nos expériences suivantes mobilisant la phase d'exploration décrite en ces pages. Nous procédons désormais ainsi : après avoir formulé la question ouverte, nous présentons la grille au sujet et lui expliquons la façon dont nous allons la remplir. Ainsi, le sujet connaît nos attentes. Mais nous ne nous servons que d'une seule grille et remplissons par la suite des feuilles vierges, c'est-à-dire sans impression de la figure 1.

- Prévoir une surface suffisante permettant éventuellement d'étaler quelques grilles (un bureau, une table). Lors d'un entretien, en phase de test de la technique, la surface trop petite (bureau fortement encombré...) nous a obligés à poser les grilles d'exploration les unes sur les autres. Le discours de l'interviewé peut conduire à passer d'une grille à l'autre. La recherche de la bonne grille, lorsqu'un changement s'impose, distrait le sujet qui a alors tendance à interrompre son énoncé.

- Maintenir, lors de la phase de validation, un rythme dans les explications ne permettant pas à la carte de jouer pleinement son pouvoir émancipatoire. C'est plus tard qu'il y a un intérêt à ce qu'il s'exprime.

\section{Conclusion}

La méthode proposée, opérationnelle et simple, peut inspirer les missions de mise en place d'un contrôle dans les jeunes entreprises et dans les TPE, voire dans certaines $\mathrm{PME}^{9}$, qui ne disposent pas de la batterie de spécialistes de la grande

9. À partir d'un certain niveau de complexité, il est nécessaire de la combiner à d'autres méthodes pour la définition des systèmes et procédures de contrôle. 
entreprise. Elle permet de sensibiliser au besoin de contrôle, de mesure des options stratégiques et de mise au point de la vision du dirigeant. À ce titre, il peut être intéressant de réitérer l'expérience avec le même individu puisque l'on sait que cette vision évolue (ce que les travaux utilisant la métaphore biologique du cycle de vie ont montré), tout comme les FCS et FSR ( $c f$. les ouvrages portant sur la stratégie ou le management stratégique). Cela peut être aussi une façon de suivre la complexité croissante de l'entreprise et de mettre en place un contrôle « harmonieux». En effet, il convient de ne pas écraser la jeune entreprise par le formalisme des procédures de gestion; outre le fait qu'elle n'en a pas les moyens, elle n'en a pas vraiment besoin.

Soulignons le caractère pédagogique de la méthode, et souhaitons son développement et son adaptation de façon à ce que le dirigeant puisse lui-même mettre au point sa vision stratégique (Cossette, 1994a) ; son caractère émancipatoire, par exemple, est une source indéniable d'apprentissage. Les propos métaphoriques de Laroche et Nioche nous aident à l'accepter: " les cartes cognitives ressemblent à un plan qu'un ami vous dessine pour atteindre sa maison de campagne. À la différence de la carte Michelin, le plan ne vous indique que l'itinéraire correct. Si vous vous en écartez, vous risquez d'être perdu en terra inconita. Il vous faudra alors péniblement apprendre à déchiffrer un territoire inconnu [...]» $(1994$, p. 69). En ce sens, répéter l'exercice présenté ici peut être un moyen de repérer l'alternative stratégique permettant à l'entreprise de retrouver le chemin des objectifs qu'elle s'est fixée, voire de les amender.

Ce travail ouvre de nombreuses voies de recherche, en voici des exemples: relever les systèmes et processus de contrôle à l'œuvre dans les entreprises naissantes ou les TPE pour en effectuer une typologie (notamment pour le repérage de la dimension informelle du contrôle), mettre au point un projet de création d'entreprise (aider le créateur potentiel à rendre plus claires ses idées, à mettre au point sa vision), identifier les FCS et FSR perçus par une catégorie d'acteurs (experts, créateurs d'entreprise, comparaisons entre dirigeants d'entreprise évoluant dans des secteurs différents, etc.), mettre en évidence l'évolution des priorités du créateur dans le temps ( $c f$. travaux sur les cycles de vie), comparer les visions stratégiques d'une catégorie d'acteurs, relever l'existence de problématiques données dans la vision du sujet (p. ex., les problématiques liées à l'emploi dans la vision du dirigeant), etc.

Ces voies sont autant de dimension opérationnelle (la méthode est alors déployée plus ou moins complètement), que de dimension fondamentale (les deux premières phases étant le préalable à une phase d'analyse adaptée à l'objet d'étude) dès lors qu'est reconnue l'utilité de relever les facteurs perçus par les acteurs (mais après tout, dans le champ des sciences sociales, ne travaille-t-on pas principalement sur des données issues de la représentation des individus interrogés?). Il est possible de reconnaître à la cartographie cognitive une 
utilité certaine dans l'identification des facteurs d'évolution des firmes (sous l'angle donc de la perception qu'ont les acteurs de ces facteurs), à condition de répéter les observations dans le temps (approche longitudinale). Elle constitue, dès lors qu'elle s'inscrit dans une démarche méthodologique rigoureuse, un outil adéquat à cette identification. Certes, l'interprétation d'une carte est le fruit d'un chercheur, mais n'est-ce pas le lot de toute recherche qualitative, et l'interprétation n'est-elle pas le lot de toute recherche de compréhension? La collecte des données (phase d'exploration et de validation) mobilise deux acteurs, le sujet et le chercheur, et le caractère circulaire des échanges tend à mettre en phase leur interprétation respective au regard de l'objet de leurs rencontres. On peut raisonnablement considérer que le chercheur a une interprétation du concept évoqué par le sujet proche de la propre interprétation de celui-ci, et cela d'autant plus qu'outre l'interrogation systématique de la première phase (exploration), la seconde phase (validation) permet de vérifier la similitude des interprétations. Le chercheur doit néanmoins obligatoirement s'astreindre à la rédaction d'un texte narratif relatif aux propos tenus par les sujets. Cela évite les approches mécanistes, favorise la cohérence, oblige l'honnêteté et les explicitations, permet des approfondissements (Huberman et Miles, 1991) et, plus évidemment, cela permet la lecture de la carte. Nous n'avons aucun mal à lire celle présentée en ces pages, M. Petit non plus. Si le but de cette communication avait été de la décrire au lecteur, un texte narratif l'aurait accompagné.

\section{Bibliographie}

Abelson, R.P et M.J. Rosenberg (1958), «Symbolic psychologic: a model of attitudinal cognition », Behavioural Sciences, $n^{\circ} 3$, p. 1-13.

ABRIC, J.C. (1984), «L'artisan et l'artisanat: analyse du contenu et de la structure d'une représentation sociale », Bulletin de psychologie, tome XXXVII, n 366 , p. 861-875.

ABRIC, J.C. (1989), «L'étude expérimentale des représentations sociales », dans Jodelet (éd.), Les Représentations Sociales, Paris, PUF.

ABRIC, J.C. (1994), Pratiques et représentations sociales, Paris, PUF.

Allard-PoEsi, F. (1995), « De schémas cognitifs individuels à un schéma cognitif collectif : vers une perspective sociocognitive», IVe Conférence internationale de management stratrégique de l'AIMS, Paris.

AllaRD-PoEsI, F. (1996), « Cartes cognitives : pour ne pas jeter le bébé avec l'eau du bain », Ve Conférence internationale de management stratrégique de l'AIMS, Lille.

ANThony, R.N. (1965), Planning and Control Systems : A Framework for Analysis, Boston, Harvard University Press. 
ARDOIN, J.L., D. MiCHEL et J. SCHMIDT (1986), Le contrôle de gestion, Paris, Publi Union.

Audet, M. (1994), «Plasticité, instrumentalité et reflexivité », dans Cossette (éd.), Cartes cognitives et organisations, Québec, Les Presses de l'Université Laval; Paris, Éd. Eska, p. 187-198.

AVENIER, M.J. (1988), Le pilotage stratégique de l'entreprise, Paris, Presses du CNRS.

Axelrod, R. (1976), The Cognitive Maps of Political Elites, Princeton, N.J., Princeton University Press.

BAumard, P. (1996), Prospective à l'usage du manager, Paris, Litec.

Beauvois, J.L. et J.C. Deschamps (1990), «Vers la cognition sociale », dans Traité de psychologie cognitive, Tome 3, Paris, Dunod, p. 1-10.

Bougon, M.G. et J.M. KomOCAR (1994), « Façonner et diriger la stratégie. Approche holistique et dynamique », dans Cossette (éd.), Cartes cognitives et organisations, Québec, Les Presses de l'Université Laval, Paris, Éd. Eska, p. 57-80.

BoUQUin, H. (1986), Le contrôle de gestion, Paris, PUF.

BourdieU, P. (1980), Le sens pratique, Paris, Édition de Minuit.

BRUYAT, C. (1993), «Création d'entreprise : contributions épistémologiques et modélisation », Thèse pour le doctorat de sciences de gestion, Grenoble.

BRUYAT, C. (1994), «Contributions épistémologiques au domaine de l'entreprenariat », Revue Française de Gestion, novembre-décembre.

BuIgues, P.A. (1985), Prospective et compétitivité, Paris, McGraw-Hill.

CossetTe, P. (1994a), «Structures cognitives et organisations », dans Louche (éd.), Individu et organisations, Paris, Delachaux et Niestlé, p. 155-178.

Cossette, P. (1994b), Cartes cognitives et organisations, Québec, Les Presses de l'Université Laval ; Paris, Éd. Eska, p. 3-12.

COSSETTE, P. (1994c), « Développement d'une méthode systématique d'aide à la mise au point de la vision stratégique chez le propriétaire dirigeant de PME : une étude exploratoire ", Colloque PME de Strasbourg, p. 73-82.

Cossette, P. et M. Audet (1994), «Qu'est-ce qu'une carte cognitive ? », dans Cossette (éd.), Cartes cognitives et organisations, Québec, Les Presses de l'Université Laval ; Paris, Éd. Eska, p. 13-36.

Crozier, M. et E. Friedberg (1977), L'acteur et le système, Paris, Seuil.

Demasio, A.R. (1995), L'erreur de Descartes, Paris, Éd. Odile Jacob.

DEGENNE, A. et M. Forsé (1994), Les réseaux sociaux, Paris, Armand Colin.

DENIS, M. (1989), Image et cognition, Paris, PUF.

Desreumaux, A. (1993), Stratégie, Paris, Dalloz. 
DORÉ, F et P. MERCIER (1992), Les fondements de l'apprentissage et de la cognition, Lille, Presses universitaires de Lille; Boucherville, Gaëtan Morin Éditeur.

FESTINGER, L (1957), A Theory of Cognitive Dissonance, New York, Harper and Row.

Fiol, M.C. et A.S. HufF (1992), «Maps for managers : where are we ? Where do we go from here ? », Journal of Management Studies, vol. 29, nº 3, mai, p. 267-286.

Godet, M. (1991a), L'avenir autrement, Paris, Armand Colin.

Godet, M.(1991b), De l'anticipation à l'action. Manuel de prospective et de stratégie, Paris, Dunod.

GRIZE, J.B. (1989), «Logique naturelle et représentations sociales », dans Jodelet (éd.), Les représentations sociales, Paris, PUF, p. 152-168.

GUIMELLI, C. (1994), « Transformation des représentations sociales : pratiques nouvelles et schèmes cognitifs de base », dans Guimelli (éd.), Structures et transformations des représentations sociales, Paris, Delachaux et Niestlé.

Hatem, F., B. Cazes et F. Roubelat (1993), La prospective. Pratiques et méthodes, Paris, Economica.

HeIDER, F (1958), The Psychology of Interpersonnal Relations, New York, Wiley.

Huberman, A.M. et M.B. Miles (1991), Analyse des données qualitatives, Bruxelles, Université De Boeck.

HufF, A.S., V. NAPPAREDDY et K.E. FLeTCHER (1990), « Coding the causal association of concepts », dans Huff (éd.), Mapping Strategic Thought, Chichester, John Wiley et Sons.

Hume, D. (1983), Enquête sur l'entendement humain, Paris, Flammarion, réédition, éd. or. 1758.

KELLEY, H.H. (1967), « Attribution theory in social psychology », dans Levine (éd.), Nebraska Symposium of Motivation.

KoEnIG, G. (1990), Management stratégique, Paris, Nathan.

LANGFIELD-SMITH, K.M. (1992), «Exploring the need for a shared cognitive map », Journal of Management Studies, vol. 29, nº 3, mai, p. 349-368.

LAROCHE, H et J.P. NIOCHE (1994), «L'approche cognitive de la stratégie d'entreprise », Revue Française de Gestion, juin-juillet-août, p. 64-78.

LAZEGA, E. (1994), « Analyse de réseau et sociologie des organisations », Revue Française de Sociologie, vol. 3.

LEIDECKER, J.K. et V. BRUNO (1984), «Identifying and using critical success factors », Long Range Planning, vol. 17, $\mathrm{n}^{\circ} 1$.

Livet, P. (1993), « Théorie de l'action et conventions », dans Quéré (éd.), La théorie de l'action. Le sujet pratique en débat, Paris, CNRS Éditions. 
MinTZBERG, H. (1994), Grandeur et décadence de la planification stratégique, Paris, Dunod.

MOLINER, P. (1994), «Les méthodes de repérage et d'identification du noyau des représentations sociales », dans Guimelli (éd.), Structures et transformations des représentations sociales, Paris, Delachaux et Niestlé, p. 199-232.

PAILHous, J. (1970), La représentation de l'espace urbain, Paris, PUF.

Piaget, J. (1967), Biologie et Connaissance, Paris, Gallimard.

Piatelli-Palmarini, M. (1995), La réforme du jugement ou comment ne plus se tromper, Paris, Odile Jacob.

SAPORTA, B. (1994), «La création d'entreprises: enjeux et perspectives », Revue Française de Gestion, novembre-décembre.

ThÉVENOT, L. (1993), « Agir avec d'autres. Conventions et objets dans l'action coordonnée », dans Quéré (éd.), La théorie de l'action. Le sujet pratique en débat, Paris, CNRS Éditions.

Tolman, E.C. (1948), «Cognitive maps in rats and men», Psychological Review, vol. 55.

VERNIER, P. et D. VINCENT (1996), «Le rôle majeur des émotions », Sciences et vie, hors série, $n^{\circ} 195$, juin.

Verstraete, T. (1995), « Cartographie cognitive et accompagnement de projet de création d'entreprise », $\mathrm{II}^{\mathrm{e}}$ Congrès international francophone de la PME, Paris.

Verstraete, T. (1996a), "Cartographie cognitive et accompagnement de projet de création d'entreprise », Cahier de Recherche du CLAREE, Lille, janvier.

Verstraete, T. (1996b), «La cartographie cognitive : outil pour une démarche d'essence heuristique d'identification des facteurs-clés de succès », Ve Conférence Internationale de Management Stratrégique, Lille.

VOGLER, E. (1995), «Management stratégique et psychologie cognitive. Un aperçu rapide de la psychologie cognitive », Cahiers de recherche, Groupe ESC Lyon, tome 1 .

VOGLER, E. (1996), « Management stratégique et psychologie cognitive. Synthèse des emprunts du management stratégique à la psychologie cognitive », Cahiers de recherche, Groupe ESC Lyon, tome 2.

WeICK, K.E. (1979), The Social Psychology of Organizing, Reading, Mass. AddisonWestley.

WEICK, K.E. et M.B. BougON (1986), « Organizations as cognitive maps : charting ways to success and failure », dans Sims et Gioia (éd.), The Thinking Organization, San Francisco, Jossey-Bass, p. 102-135. 